\title{
Beyond TNM: Searching for New Patient-Centric Prognostic Indicators in NSCLC
}

\author{
Areo G. Saffarzadeh, MD and Justin D. Blasberg, MD \\ Yale New Haven Hospital, New Haven, CT
}

The mainstay of treatment for early-stage non-small cell lung cancer (NSCLC) in suitable operative candidates is anatomic resection. ${ }^{1}$ For patients and providers, prognostication is an essential component of developing an individualized treatment plan. TNM stage at presentation is an important indicator of prognosis, and the recently updated 8th edition of the lung cancer staging guidelines further refines subcategories of stage I cancer (IA1, IA2, IA3), providing more granularity to estimate 5-year survival. $^{2}$ However, utilizing clinical or pathologic stage as a predictor of 5-year survival is not without limitations. Clinical stage provides just a single snapshot of tumor burden, and the TNM system is predominately intended to provide a common nomenclature to describe the anatomic extent of tumor. While clinical stage accounts for $65 \%$ of variability in 5-year survival, pathologic stage accounts for less than half. ${ }^{3}$ What this means is that in surgically treated lung cancers, prognosis is driven primarily by other factors. Beyond TNM staging, we currently have only a handful of clinical parameters that help prognosticate overall survival. These can broadly be divided into two categories: (1) parameters based on the tumor, and (2) parameters based on the patient.

Parameters based on the tumor that affect prognosis include: metabolic activity seen on PET scan, histologic grade, and more recently molecular/genetic characterizations (some of which indicate responsiveness to targeted therapies such as EGFR, ALK rearrangements, and PDL1). ${ }^{4-6}$ This is an exciting area of ongoing research, and the National Cancer Institute's ALCHEMIST trials are

\section{(C) Society of Surgical Oncology 2018}

First Received: 8 August 2018;

Published Online: 28 August 2018

J. D. Blasberg, MD

e-mail: Justin.blasberg@yale.edu exploring the role of targeted therapies in early stage NSCLC. ${ }^{7}$ Prognostic parameters based on the patient often are utilized by surgeons to determine operative candidacy. These include pulmonary function tests, comorbidities, and performance status. ${ }^{8}$ Previous work has demonstrated that patient comorbidities and reduced performance status are associated with decreased survival. ${ }^{9-11}$

Moving beyond TNM staging, recent research has focused on identifying additional patient-centric prognostic indicators of survival, specifically examining host response to tumor by inflammatory mediators and changes in the hematologic system. ${ }^{12,13}$ The link between inflammation and cancer is well established. ${ }^{14,15}$ However, the prognostic utility of easily obtainable lab values, such as neutrophil-to-lymphocyte ratio (NLR), platelet-to-lymphocyte ratio, and CRP/albumin ratio in NSCLC, is an area of active research. ${ }^{12,16}$

The study presented by $\mathrm{Li}$ et al. identifies a new prognostic indicator for NSCLC based on a serum albumin and globulin levels. ${ }^{17}$ While the importance of albumin as a marker of nutritional status has been previously established, only recently has a link between serum albumin and cancer-specific survival been defined. ${ }^{13,18}$ Proinflammatory cytokines that promote tumor development also suppress production of albumin by hepatocytes, contributing to hypoalbuminemia. ${ }^{19}$ Globulin represents the remainder of nonalbumin protein measured in serum and includes acute phase reactants and other proinflammatory mediators. Li's study defines the albumin-globulin score (AGS) and demonstrates that such a measurement not only correlates with clinical stage but also serves as an independent predictor of disease-free survival (DFS) and overall survival (OS) in NSCLC patients. While the initial analysis was completed in a retrospective fashion $(n=512)$, the authors validated their findings in a small prospective cohort $(n=53)$ to help define the clinical utility of the AGS. 
If these finding are validated by other prospective studies, the AGS score may represent an important step beyond TNM stage for prognostication in operable NSCLC. Further research is needed to determine the utility of incorporating the AGS score and other patient-centric prognostic parameters with TNM stage to inform clinical decision making. Such information also may help to define specific patient subgroups that most benefit from adjuvant therapies. The work by $\mathrm{Li}$ and others represents the frontier of thoracic oncology and is likely to have a significant clinical impact as this field moves towards understanding higher levels of complexity within the tumor biology space. Future prognostic work may complement Li's study and help define clinically important tools to define patients are at risk for poor outcomes after treatment for NSCLC.

DISCLOSURE This work does not contain data on human subjects research and has been exempted by the Yale University IRB.

\section{REFERENCES}

1. Howington JA, Blum MG, Chang AC, Balekian AA, Murthy SC. Treatment of stage I and II non-small cell lung cancer: Diagnosis and management of lung cancer, 3rd ed: American College of Chest Physicians evidence-based clinical practice guidelines. Chest. 2013;143(5 Suppl):e278S-313S. https://doi.org/10.1378/c hest.12-2359.

2. Detterbeck FC, Boffa DJ, Kim AW, Tanoue LT. The eighth edition lung cancer stage classification. Chest. 2017;151(1):193-203. https://doi.org/10.1016/j.chest.2016.10. 010.

3. Detterbeck FC, Chansky K, Groome P, et al. The IASLC lung cancer staging project: methodology and validation used in the development of proposals for revision of the stage classification of NSCLC in the forthcoming (eighth) edition of the TNM classification of lung cancer. J Thorac Oncol. 2016;11(9):143346. https://doi.org/10.1016/j.jtho.2016.06.028.

4. Nair VS, Krupitskaya Y, Gould MK. Positron emission tomography $18 \mathrm{~F}$-fluorodeoxyglucose uptake and prognosis in patients with surgically treated, stage I non-small cell lung cancer: a systematic review. J Thorac Oncol. 2009;4(12):1473-9. https://d oi.org/10.1097/jto.0b013e3181bccbc6.

5. Sun Z, Aubry M-C, Deschamps C, et al. Histologic grade is an independent prognostic factor for survival in non-small cell lung cancer: an analysis of 5018 hospital- and 712 population-based cases. J Thorac Cardiovasc Surg. 2006;131(5):1014-20. https://d oi.org/10.1016/j.jtcvs.2005.12.057.

6. D'Angelo SP, Janjigian YY, Ahye N, et al. Distinct clinical course of EGFR-mutant resected lung cancers: results of testing of 1118 surgical specimens and effects of adjuvant gefitinib and erlotinib. J Thorac Oncol. 2012;7(12):1815-22. https://doi.org/ 10.1097/jto.0b013e31826bb7b2.

7. Govindan R, Mandrekar SJ, Gerber DE, et al. ALCHEMIST trials: a golden opportunity to transform outcomes in early-stage non-small cell lung cancer. Clin Cancer Res. 2015;21(24):5439-44. https://doi.org/10.1158/1078-0432.ccr-150354.

8. Brunelli A, Kim AW, Berger KI, Addrizzo-Harris DJ. Physiologic evaluation of the patient with lung cancer being considered for resectional surgery: diagnosis and management of lung cancer, 3rd ed: American college of chest physicians evidence-based clinical practice guidelines. Chest. 2013;143(5 Suppl). https://doi. org/10.1378/chest.12-2395.

9. Battafarano RJ, Piccirillo JF, Meyers BF, et al. Impact of comorbidity on survival after surgical resection in patients with stage I non-small cell lung cancer. J Thorac Cardiovasc Surg. 2002;123(2):280-7. http://www.ncbi.nlm.nih.gov/pubmed/11828 287. Accessed 2 Aug 2018.

10. Kawaguchi T, Takada M, Kubo A, et al. Performance status and smoking status are independent favorable prognostic factors for survival in non-small cell lung cancer: a comprehensive analysis of 26,957 patients with NSCLC. $J$ Thorac Oncol. 2010;5(5):620-30. https://doi.org/10.1097/jto.0b013e3181d2dcd 9.

11. Buccheri G, Ferrigno D. Cancer MT-EJ of 1996 undefined. Karnofsky and ECOG performance status scoring in lung cancer: a prospective, longitudinal study of 536 patients from a single institution. Elsevier. https://www.sciencedirect.com/science/artic le/pii/0959804995006648. Accessed 2 Aug 2018.

12. Zhang H, Xia H, Zhang L, Zhang B, Yue D, Wang C. Clinical significance of preoperative neutrophil-lymphocyte vs plateletlymphocyte ratio in primary operable patients with non-small cell lung cancer. Am J Surg. 2015;210(3):526-35. https://doi.org/10. 1016/j.amjsurg.2015.03.022.

13. Shibutani $M$, Maeda $K$, Nagahara $H$, et al. The pretreatment albumin to globulin ratio predicts chemotherapeutic outcomes in patients with unresectable metastatic colorectal cancer. BMC Cancer. 2015;15(1):347. https://doi.org/10.1186/s12885-015-137 5-x.

14. Mantovani A, Allavena P, Sica A, Balkwill F. Cancer-related inflammation. Nature. 2008;454(7203):436-44. https://doi.org/1 $0.1038 /$ nature 07205 .

15. Coussens LM, Werb Z. Inflammation and cancer. Nature. 2002;420(6917):860-7. https://doi.org/10.1038/nature01322.

16. Koh YW, Lee HW. Prognostic impact of C-reactive protein/albumin ratio on the overall survival of patients with advanced nonsmall cell lung cancers receiving palliative chemotherapy. Med. 2017;96(19):e6848-8. https://www.ncbi.nlm.nih.gov/pmc/a rticles/PMC5428608/. Accessed Aug 22018.

17. Li, Qin, Sun, et al. Prognostic significance of albumin-globulin score in patients with operable non-small-cell lung cancer. Ann Surg Oncol. 2018 (in press).

18. Gupta D, Lis CG. Pretreatment serum albumin as a predictor of cancer survival: a systematic review of the epidemiological literature. Nutr J. 2010;9(1) 5-9. https://doi.org/10.1186/14752891-9-69.

19. Chojkier M. Inhibition of albumin synthesis in chronic diseases: molecular mechanisms. J Clin Gastroenterol. 2005;39(4 Suppl 2):S143-6. https://doi.org/10.1097/01.mcg.0000155514.17715. 39. 